$\begin{array}{ll}\text { le portiQue } & \text { Le Portique } \\ \text { Revue de philosophie et de sciences humaines }\end{array}$

$18 \mid 2006$

Heidegger. La pensée à l'heure de la mondialisation

\title{
Heidegger en Iran
}

\section{Daryush Shayegan}

\section{OpenEdition}

\section{Journals}

Édition électronique

URL : https://journals.openedition.org/leportique/817

DOI : 10.4000/leportique.817

ISSN : $1777-5280$

\section{Éditeur}

Association "Les Amis du Portique"

Édition imprimée

Date de publication : 1 septembre 2006

ISSN : 1283-8594

Référence électronique

Daryush Shayegan, « Heidegger en Iran », Le Portique [En ligne], 18 | 2006, mis en ligne le 15 juin 2009, consulté le 20 février 2023. URL : http://journals.openedition.org/leportique/817 ; DOI : https://doi.org/ 10.4000/leportique.817

Ce document a été généré automatiquement le 20 février 2023.

Tous droits réservés 


\section{Heidegger en Iran}

\section{Daryush Shayegan}

1 Heidegger a connu en Iran, surtout dans les années soixante-dix, une très grande vogue. C'est à cette époque qu'on vit surgir le fameux groupe des heideggériens islamisants qui, après la révolution, non seulement ne disparut pas de la scène culturelle du pays, mais au contraire, la république islamique aidant, devint même plus puissant.

2 Mais pour comprendre la nature de ce courant de pensée qui perdure toujours et qui a revêtu à présent un masque très réactionnaire, il convient, à mon avis, de cerner le personnage étrange de son fondateur Ahmad Fardid (1912-1994). J'ai connu personnellement Fardid. On se réunissait régulièrement avec Fardid et les autres chez notre ami Hossein Jahanbegloo; ces soirées, en raison de son animateur, furent baptisées Fardidiyeh. C'était un laboratoire in vivo de tous les conflits qui, à l'époque, déchiraient les esprits: conflits entre modernité et tradition, absolutisme et démocratie, libéralisme et communisme. Fardid se référait constamment aux positions extrêmes du Maître de Freiburg, utilisait sans gêne ses idées à ses fins particulières et en tiraient des conclusions vertigineuses. Tout ce que je me rappelle de ces soirées agitées, théâtrales, souvent cocasses à la limite du ridicule, ce sont les accrochages, les affrontements verbaux, les voltes-faces scandaleuses. C'étaient des soirées enrichissantes et déprimantes à la fois. Fardid était un provocateur-né ; il avait, sans aucun doute, des éclairs de génie et des intuitions fulgurantes qui vous laissaient pantois. Il était une sorte de chaman-philosophe qui, fidèle à tradition socratique, ne daignait jamais coucher une ligne sur le papier de peur qu'on ne trahisse sa pensée. Il était, d'autre part, incapable de tenir une démonstration cohérente; à peine s'était-il embarqué dans une idée que d'autres jaillissaient de plus belle et déviaient son discours pour aboutir parfois à de méchantes et perfides insinuations. Les idées étincelantes, incendiaires, s'étouffaient dans un magma de ressentiment, de concepts à peine ébauchés, d'idées avortées et le tout sur la toile de fond d'un immense désir d'interpréter toute l'histoire intellectuelle de l'Occident à la lumière de son exégèse personnelle de quelques concepts fondamentaux de Heidegger: la dispensation et l'oubli de l'Être (Seinsvergessenheit). 
Que l'histoire de l'Être soit les étapes « historiales » d'une vérité qui se décline, ou qu'il $\mathrm{y}$ ait retrait de l'Être et son oubli, voilà qui arrangeait fort bien l'esprit mystique de Fardid, pour qui tout mouvement progressif dans l'histoire, tout progrès de l'homme, tout acquis des Lumières, n'étaient, en dernier ressort, que le travail prodigieux du négatif, voire les masques du nihilisme. Car, à vrai dire, l'occultation d'un principe qui recule en dévoilant telle vérité à telle époque, et l'engloutissement d'un monde qui s'enlise dans les ténèbres du non-être, s'accommodaient fort bien avec l'esprit manichéen de Fardid. C'est axé sur cette vision ontologique que Fardid interprétait d'une part, et ceci à sa façon, l'histoire de la philosophie occidentale et, d'autre part, afin d'étayer sa vision et lui trouver des précédents exemplaires dans la mystique de l'islam, puisait soit dans les grands textes du soufisme comme ceux de Ibn Arabi, par exemple, soit s'inspirait largement des poèmes ô tellement paradoxaux de Hafez de Chiraz. On assistait de la sorte à des amalgames ahurissants, à la confusion des genres et des contextes, comme si on effaçait d'un revers de la main les clivages historiques, les ruptures épistémologiques, ainsi mystique et philosophie se rejoignaient, se contaminaient pour créer Dieu sait quel étrange mélange. Sa manière de penser souffrait d'une double carence : 1. l'« identification en chaîne » (signalée par Raymond Aron dans L'Opium des intellectuels), puisque Fardid identifiait les notions linguistiques et philosophiques incommensurables les unes avec les autres et 2. la critique par extrapolation. C'est-à-dire qu'il appliquait au décryptage de son propre monde, dépaysé, décalé par rapport à l'Occident, des outils conceptuels qui, eux, appartenaient à un tout un autre contexte. Par exemple, les critiques de Heidegger sur la technique concernent la genèse de la pensée occidentale, ses mises en retrait successives à partir de la physis des présocratiques jusqu'à la volonté de puissance chez Nietzsche en passant par l'idée platonicienne, la substantialité scholastique médiévale, la subjectivité cartésienne et la dialectique de Hegel. Tout ceci reste, il va sans dire, en dehors de l'orbite de la tradition où puisait Fardid, laquelle s'affiliait en outre à une épistémê complètement différente.

4 Fardid et plus tard ses disciples franchement médiocres n'avaient cure de ses divergences essentielles. Ce qui fascinait Fardid c'était la fin de la métaphysique, et l'idée inattendue émise par Heidegger dans son interview avec l'hebdomadaire Spiegel que seul un Dieu peut nous sauver (nur ein Gott kann uns retten). Pour Fardid, toute la production littéraire, artistique de la Perse moderne étaient les déchets et les sousproduits de la «métaphysique» occidentale. Si donc on a atteint la fin de la métaphysique et que nous attendons tous l'apparition imminente d'une nouvelle divinité, quelle sera alors la configuration du monde lorsque cette même divinité finira par se révéler? Alors, disait emphatiquement Fardid, le point final de l'«aprèsdemain » (pas fardâ) de l'Être rejoindra inéluctablement le point initial de son « avanthier » (pari rouz), c'est-à-dire de son commencement. Par conséquent la boucle serait bouclée et le messianisme contenu dans le message sotériologique de l'Être trouverait son achèvement et sa consomption dans sa fin même, voire dans l'éclosion initiale de sa Vérité.

5 On trouve ainsi chez Fardid heideggérien deux éléments concomitants: 1. la mythologisation de la pensée et 2 . le contenu messianique d'une promesse eschatologique qui pour un manichéen invétéré comme Fardid, et de surcroît iranien, est une aubaine inespérée, car il peut de la sorte "moderniser", grâce à l'herméneutique amplifiante du philosophe allemand, une vision messianique aussi 
vieille que le monde. Car n'oublions pas que dans l'arrière-fond de sa pensée, l'idée de messianisme qui est profondément ancrée dans la mémoire collective iranienne, est très présente. Adorno avait déjà perçu dans la vision de Heidegger le danger de la mythologisation de la pensée. Voici ce qu'il dit: «l'histoire du penser est, aussi loin qu'on puisse le suivre, dialectique de l'Aufklärung. C'est pourquoi Heidegger, de façon assez résolue, ne s'arrête pas, comme cela aurait pu le séduire dans sa jeunesse, à une quelconque de ces étapes, mais se précipite avec une machine à remonter le temps à la Wells, dans l'abîme de l'archaïsme dans lequel tout peut être tout et tout signifier. Il tend la main au mythe; mais même le sien reste un mythe du $\mathrm{xx}^{\mathrm{e}}$ siècle, illusion, comme l'histoire l'a démasqué et qui devient éclatante par le caractère absolument inconciliable du mythe avec la figure rationalisée de la réalité dans laquelle toute conscience est prise ${ }^{1}$.

6 C'est donc en s'inspirant du temps de l'attente que Fardid anticipe en quelque sorte l'avènement de cette divinité attendue à l'horizon, en faisant coïncider l'«aprèsdemain » du cycle de l'Être avec son « avant-hier ». Ce concept-là, on le retrouve aussi bien dans la pensé chiite que dans l'ancienne religion zoroastrienne de l'Iran. Si Heidegger parle d'un Dieu qui viendra peut-être nous sauver, Fardid y adhère sans condition, car la fin d'un cycle n'est-il pas, tout compte fait, une transfiguration du monde? Que la pensée iranienne se soit sentie si familière dans le climat messianique du chiisme, cela n'est point une surprise. Tout le cycle des douze millénaires de la cosmologie zoroastrienne aboutissant à la Transfiguration finale du monde l'y prédisposait déjà. Sans doute les formes avaient changé, sans doute le cadre religieux s'était déplacé vers le cycles des religions abrahamiques mais le message profond d'une Transfiguration du monde vécue intensément par la conscience iranienne - si enracinée dans l'idée d'exil de l'homme en ce monde est resté intact, et Fardid n'y échappa pas non plus, il trouva chez Heidegger une justification philosophique moderne pour son obsession métaphysique.

7 Après la révolution, les choses changèrent de fond en comble. Fardid, en opportuniste consommé, tourna casaque et devint un révolutionnaire illuminé presqu'enragé. Peutêtre que dans sa pensée l'après-demain avait-il rejoint d'ores et déjà l'avant-hier de l'Être, peut-être la divinité qu'il attendait si impatiemment avait-elle revêtu la figure hiératique du Guide suprême. Et tout comme pour son Maître, le Führer s'incarnait dans le Geist rénovateur du Volk allemand, de même pour Fardid, le Sauveur prenait la figure du Mage qui venait instaurer un Ordre nouveau. Avec la révolution, Fardid se métamorphosa en un prophète, un visionnaire de l'âge nouveau, un voyant qui, grâce à l'attraction magnétique que lui accordait le nouvel ordre spirituel, devint la figure charismatique de toute une jeune génération religieuse, subjuguée par cet événement rare, envoûtée par la magie d'un grand ébranlement qui n'est plus une lutte de classes au sens classique du terme, mais l'instauration sans précédent d'une utopie messianique. Cette génération était surtout soucieuse de trouver un alibi moderne à même de la relier à l'histoire du monde et Fardid allait lui en fournir.

8 Dans la première conférence publique qu'il prononça à l'université de Téhéran après le triomphe de la révolution et à laquelle j'assistais, il donna le ton de ce nouvel esprit. Je me souviens distinctement d'une seule phrase qui me fit froid dans le dos. Il dit - et je cite de mémoire - «les droits de l'homme - donc les acquis des Lumières - sont une censure politique qu'exerce l'infra-conscience (al-nafs al-ammâra) sur la supraconscience, c'est-à-dire l'âme pacifiée (al-nafs al motma'yanna) ». Je me souviens très 
bien de ce que je lui ai dit en sortant: «vous avez signé l'arrêt de mort de tous les intellectuels laïques». Et ce fut la dernière fois que je l'ai vu. Je ne pouvais pas comprendre qu'un homme se disant philosophe-sage eût pu cautionner les exécutions sommaires et inaugurer à sa façon le règne de la terreur. Dès lors il n'est guère étonnant que cette pensée devenue meurtrière, dangereuse, obscurantiste ait attiré la meute radicale des jeunes enragés à la recherche d'un discours moderne quitte à justifier la violence du sacré qui faisait irruption sur la scène politique. Et ce n'est pas étonnant non plus que Fardid ait influencé surtout les naïfs, les paumés révolutionnaires, les non-versés dans la culture occidentale, les idéologues endurcis par cette nouvelle vision messianique du monde qui faisaient du pays un champ de bataille apocalyptique. Non seulement les islamistes de tout acabit coururent en rangs serrés vers ce fardidisme heideggérien, non seulement la censure trouva son apothéose dans la victimisation de l'âme pacifiée et sublime, non seulement tout ce qui relevait des Lumières et des droits de l'homme étaient relégués au rang des aberrations sataniques, mais il y eût même des intellectuels respectables et sérieux qui suivirent cet appel. J'y reviendrai. Il se créa ainsi, au long des années, un réseau presque mafieux autour de son charisme. Un réseau qui s'infiltra dans les appareils de l'État, dans les médias, dans la presse et donna naissance à tout un cortège sinistre de censeurs, d'inquisiteurs qui, à l'affût des «libéraux déviants ", ne cherchaient qu'à les dénoncer, les traîner dans la boue, les compromettre et même causer leur perte. La délation intellectuelle qui a cours même aujourd'hui demeure en grande partie leur œuvre.

Pour en revenir aux intellectuels sérieux, un exemple parmi d'autres, je cite le cas d'un philosophe, fardidien et heideggérien de première heure, qui, parlant de l'art occidental, nous dit à peu près ceci : l'art de l'Occident est un art satanique (shaytani), car la vérité s'y voile sous forme du moi, de l'orgueil, de l'infra-conscience (nafs). L'art futur est, en revanche, un art miséricordieux (rahmani). C'est l'ère de la sym-pathie, du dia-logue, de la contemplation directe. Mais quand la religion entre en exil et que la Divinité se retire, alors les artistes et les poètes en particulier s'évertuent à renouer L'Alliance (tajdid-e 'ahd), à ressusciter le sacré. Ce renouvellement est l'instauration de la République islamique. Je disais quelque part à ce sujet: "tout le raisonnement sublime de notre philosophe repose sur une interprétation heideggérienne de la vérité. Il fallait en somme qu'un philosophe allemand du $\mathrm{xx}^{\mathrm{e}}$ siècle se penchât sur les étapes historiales de la philosophie occidentale, qu'il l'interprétât dans le sens de l'Occultation de l'Être, qu'il en dévoilât, à sa manière, les différents discours dans le temps, pour qu'un Iranien, situé aux antipodes de ce monde, en prît connaissance, grâce aux traductions françaises, et qu'il s'estimât concerné par un problème qui n'est d'ailleurs pas le sien; et, pour comble d'illusion, crût déceler dans l'annonce messianique de l'Allemand la vérité spirituelle du renouveau islamique ${ }^{2}$.

Pour conclure je dirais que Heidegger, perçu par la grille messianique de Fardid et repris plus tard par la bande de ses disciples qui ne cessent de célébrer son culte pour en faire un personnage quasi prophétique, est un mal qui ronge de l'intérieur l'esprit critique et toute la tradition des Lumières dont les pays comme les nôtres ont tant besoin. Si Fardid et beaucoup d'autres qui l'ont suivi ont pu interpréter Heidegger de cette manière, c'est parce que, comme le dit si bien Adorno, la pensée de ce dernier, en vertu de sa dimension mythique, s'y prêtait à merveille. En d'autres termes je pense que la pensée de Heidegger, en dépit de ses immenses mérites, se livre mieux à ces amalgames que celle plus critique, plus sereine de Kant. J'estime que, pour les traditions comme les nôtres, où la pensée critique a tant de mal à prendre pied, la 
philosophie de Kant est plus «hygiénique » mentalement que celle d'autres penseurs. Tout d'abord Kant nous empêche de délirer, ensuite il montre les limites de la connaissance. Kant montre aussi que la raison ne peut s'aventurer au-delà de certaines limites sans s'enliser dans des phantasmes, c'est-à-dire sans aboutir à des antinomies insolubles. Par conséquent, la philosophie ne connaît aucune faculté supérieure à l'entendement (Verstand) et à la raison (Vernunft).

\section{NOTES}

1.. Théodor W. ADORNO, Dialectique négative, Paris, Payot, 1978, p. 99.

2.. Daryush SHAYEGAN, Le Regard motile, Éditions de l'Aube, 2002, pour l'édition de poche.

\section{RÉSUMÉS}

« Heidegger en Iran»

Heidegger, interprété par la grille messianique de Ahmad Fardid (1912-1994), fondateur en Iran $\mathrm{du}$ groupe des heideggeriens islamisants et par les disciples de ce dernier qui voient dans leur gourou un personnage prophétique incarne un mal qui paralyse tout esprit critique et rejette dans les ténèbres la tradition des Lumières dont nous avons tant besoin aujourd'hui.

"Heidegger in Iran"

Heidegger, as seen through Ahmad Fardid's messianic grid (1912-1994), who founded in Iran the group of Islamist Heideggerians and seen also through the latter's disciples who see in their guru a prophetic person, is the incarnation of a disease that paralyses all critical spirit and eclipses the tradition of the Enlightenment that we so much need these days.

“Heidegger en Irán"

Heidegger, cuando lo interpretan los discípulos de Ahman Farid (1912-1994), quien fundó en Irán el grupo de los heideggerianos islamizantes gracias a la clave mesiánica de su gurú, al que consideran como personaje profético, llega a incarnar un mal que paraliza todo espíritu crítico y arroja a las tinieblas la tradición del siglo de las luces que tanto necesitamos hoy.

"Heidegger im Iran"

Wenn Heidegger durch den durch den Messianismus eines Ahman Farid (1912-1994) oder durch seine Schüler, die islamischen Heideggerianer, entschlüsselt wird, so entwickelt er sich zum Symbol eines Übels, das jeden kritischen Geist lähmt und die Tradition der Aufklärung, die wir zur zeit so benötigen, in eine dunkle Vergangenheit verwirft. 


\section{AUTEUR}

\section{DARYUSH SHAYEGAN}

Daryush Shayegan est philosophe, indianiste et romancier. Il a notamment publié Le Regard mutilé (1989), Qu'est-ce qu'une révolution religieuse (1991), Les Illusions de l'identité (1992), Hindousime et soufisme (1997), La Lumière vient de l'Occident (2001) et Terre de mirages (2004). 